\title{
Cytokines and Chemokines in Neuropsychiatric Syndromes of Systemic Lupus Erythematosus
}

\author{
Hiroshi Okamoto, ${ }^{1,2}$ Akiko Kobayashi, ${ }^{1,2}$ and Hisashi Yamanaka ${ }^{1}$ \\ ${ }^{1}$ Institute of Rheumatology, Tokyo Women's Medical University, Tokyo 162-0054, Japan \\ ${ }^{2}$ Minami-Otsuka Institute of Technology, Minami-Otsuka Clinic, Tokyo 170-0005, Japan
}

Correspondence should be addressed to Hiroshi Okamoto, hokamoto@parkcity.ne.jp

Received 9 February 2010; Accepted 21 May 2010

Academic Editor: Brian Poole

Copyright (C) 2010 Hiroshi Okamoto et al. This is an open access article distributed under the Creative Commons Attribution License, which permits unrestricted use, distribution, and reproduction in any medium, provided the original work is properly cited.

\begin{abstract}
Neuropsychiatric syndromes of systemic lupus erythematosus (NPSLE) is a life-threatening disorder and early diagnosis and proper treatment are critical in the management of this neuropsychiatric manifestations in lupus. Brain magnetic resonance imaging (MRI), electroencephalogram (EEG), neuropsychological tests, and lumbar puncture are clinical used for the diagnosis of NPSLE. In addition to these tests, cytokine and chemokine levels in CSF have been reported as useful diagnostic marker of NPSLE. Based on the number of recently published studies, this review overviewed the roles of cytokines and chemokines in NPSLE.
\end{abstract}

\section{Introduction}

Systemic lupus erythematosus (SLE) is an autoimmune disease characterized by widespread immunologic abnormalities and multiorgan involvement, including the skin, joints, and kidney, as well as the peripheral and central nervous systems (CNS). Neuropsychiatric syndromes of systemic lupus erythematosus (NPSLE) may occur at any time during the course of the disease, and symptoms are extremely diverse, ranging from depression, psychosis, and seizures to stroke [1]. The origin of minor clinical symptoms, such as headaches and mood swings are not specific for NPSLE. In fact, SLE patients may be under the influence of other conditions capable of causing neuropsychiatric symptoms, such as infections, severe hypertensions, metabolic complications, steroid psychosis, and other drug toxicities [2]. Without proper treatment, neuropsychiatric involvement in SLE is known to increase morbidity and mortality, and therefore, the availability of beneficial treatments increases the need for the early recognition of neuropsychiatric manifestations in lupus. Along with more specific diagnostic tools and an effective method of monitoring disease activity, therapeutic responses are crucial in the management of NPSLE. Currently, tests for diagnosing NPSLE include brain magnetic resonance imaging (MRI), electroencephalogram (EEG), neuropsychological tests, and lumbar puncture. These findings are reported to be abnormal in some but not all patients, and therefore, none of the findings are specific for NPSLE. The large discrepancy in thereported frequency of neuropsychiatric involvement in SLE patients (14\%-75\%) further proves there is no single confirmatory diagnostic tool $[3,4]$. Cytokines, small substances secreted by specific cells of the immune system which carry signals locally between cells, have important roles in the development and functioning of both the innate and adaptive immune response. Chemokines are chemoattractant cytokines which play key roles in the accumulation of inflammatory cells at the site of inflammation. Increased levels of proinflammatory cytokines and chemokines have been reported in the cerebral spinal fluids (CSF) of patients with NPSLE, and some reports have shown cytokines such as interleukin-6 (IL-6), IL-1, IL-8, IL-10, tumor necrosis factor (TNF)- $\alpha$, interferon (IFN)- $\gamma$, monocyte chemotactic protein 1 (MCP-1)/CCL2, Interferon-gamma inducible protein-10 (IP-10)/CXCL10 and Fractalkine/CX3CL1 to be elevated intrathecally, thereby allowing these cytokines to be used as diagnostic tools [5-9].

Cytokines and chemokines are considered to be therapeutic targets in several chronic inflammatory disorders 
such as SLE. Based on the number of recently published studies, this review focuses on the cytokines and chemokines as biomarkers as well as pathogenic factors in NPSLE.

\section{Cytokines}

The role of TNF- $\alpha$ in lupus is still controversial. TNF- $\alpha$ may be protective in patients with lupus, since low TNF- $\alpha$ activity is associated with increased disease activity. In some patients with rheumatoid arthritis who were treated with anti- TNF$\alpha$ antibodies, antidouble-stranded DNA antibodies were detected and lupus developed in a few of these patients. By contrast, TNF- $\alpha$ may promote the pathogenesis of lupus, since the level of TNF- $\alpha$ messenger RNA was high in kidneybiopsy specimens from patients with lupus nephritis and there is a report showing that giving the anti- TNF- $\alpha$ antibody agent, infliximab, to six patients with lupus led to resolution of joint swelling in three patients with arthritis and a $60 \%$ reduction of urinary protein loss in four patients with renal lupus $[10,11]$. Serum levels of interleukin-10 (IL10) are consistently high in patients with lupus, and they correlate with the activity of the disease. IL-10 has a number of biologic effects, including stimulation of polyclonal populations of B lymphocytes. Blocking this cytokine could reduce the production of pathogenic autoantibodies [11]. Serum levels of interferon- $\alpha$ (IFN- $\alpha$ ) are also elevated in patients with active lupus and microarray studies showed that 13 genes regulated by IFN were upregulated in peripheral-blood mononuclear cells from patients with lupus, as compared with healthy controls [11].

Several cytokines such as IL-4, IL-6, IL-8, IL-10, TNF- $\alpha$, IFN- $\alpha$, and IFN- $\gamma$ have been reported to be elevated in the cerebrospinal fluid (CSF) from patients with NPSLE.

2.1. As Biomarkers. Among reported cytokines, IL-6 has been shown to have the strongest positive association with NPSLE. IL-6 level in the CSF of NPSLE was reported to be elevated without damage of the blood-brain barrier. In addition, the expression of IL- 6 mRNA was elevated in the hippocampus and cerebral cortex, suggesting that IL-6 expression was increased within the entire CNS of NPSLE $[16,17]$. There is a report studying the expression of IL-4, IL10 TNF- $\alpha$, and IFN- $\gamma$ in both peripheral blood lymphocytes (PBLs) and CSF from NPSLE patients whereby the authors found that mRNA for IL-10, TNF-a, and IFN-g were increased in PBLs while only IL-10 and IFN- $\gamma$ were elevated in CSF [6]. An exhaustive study of cytokines and chemokines recently reported that IL-6 and IL-8 were elevated in NPSLE compared with non-NPSLE and nonautoimmune disease patients. This study also found that IL-2, IL-4, IL-10, TNF- $\alpha$, and IFN- $\gamma$ were low in all groups examined [21]. In other reports, no association was found between IL-2, IL-6, IL10 , TNF- $\alpha$, and IFN- $\gamma$ with NPSLE $[13,14]$. A recent study has shown that the sensitivity and specificity of CSF IL6 for diagnosis of lupus psychosis was $87.5 \%$ and $92.3 \%$, respectively, indicating that CSF IL- 6 might be an effective marker for the diagnosis of lupus psychosis [26]. The TNF family ligands BAFF (B-cell activating factor of TNF family) and APRIL (a proliferation-inducing ligand) are essential for B-cell survival and function. Elevated serum levels of BAFF and APRIL have been reported in patients with systemic lupus erythematosus (SLE). Recently BAFF and APRIL were studied in CSF of NPSLE patients. They found that levels of APRIL in CSF were more than 20-fold higher and levels of BAFF in CSF that were more than 200-fold higher than those of healthy controls. Comparing the levels of APRIL in CSF between NPSLE and non-NPSLE patients, enhanced levels of APRIL were noted in NPSLE. Moreover, they found that CSF levels of APRIL correlated with BAFF but not with IL6 [27]. There is a report regarding the association between cytokine levels and acute confusional state (ACS) of NPSLE. The authors performed a prospective study using a cohort of 59 patients with SLE and compared those with and without ACS as well as associations between ACS and each CSF test [IL-6, IL-8, IFN- $\alpha$, IgG index, and Q-albumin]. In this study, ACS was diagnosed in 10 patients (ACS group), SLE-related CNS syndromes except ACS in 13, and no CNS syndromes in 36 (non-CNS group). CSF IL-6 levels in the ACS group were significantly higher than those in the non-CNS group $(P<$ $.05)$ and a positive IgG index $(P=.028)$ was significantly associated with ACS. No other test showed a significant association with ACS. The positive and negative predictive values for the diagnosis of ACS in SLE were $80 \%$ and $85 \%$ for elevated CSF IL-6 levels ( $>$ or $=31.8 \mathrm{pg} / \mathrm{mL}$ ), and $75 \%$ and $83 \%$ for the IgG index, respectively. From these results, the authors concluded that no single CSF test had sufficient predictive value to diagnose ACS in SLE, although CSF IL-6 levels and the IgG index showed statistical associations with ACS [28].

A summary of the reported results is shown in Table 1.

2.2. As Pathogenic Factors. Although some cytokines are important biomarkers of NPSLE, the mechanism for the elevated levels ofcytokines is thus far unknown. As SLE is an autoimmune disorder characterized by numerous autoantibodies, a pathogenetic role for autoantibodies is theoretically suspected. Immune complexes in SLE can stimulate IFN- $\alpha$ and there is strong evidence in humans and in mice that IFN$\alpha$ can cause neuropsychiatric manifestations. Santer DM et al. used a bioassay containing plasmacytoid dendritic cells to demonstrate that NPSLE CSF induced significantly higher IFN- $\alpha$ compared with CSF from patients with multiple sclerosis or other autoimmune disease controls. When normalized for IgG concentration, NPSLE CSF was 800fold more potent at inducing IFN- $\alpha$ compared with paired serum due to inhibitors present in serum. In addition to IFN- $\alpha$, immune complexes formed by CSF autoantibodies produced significantly increased levels of IP-10/CXCL, IL8, and MCP-1. From these results they proposed a twostep model of NPSLE whereby CSF autoantibodies bind to antigens released by neurocytotoxic antibodies or other brain cell injury, and the resulting immune complexes stimulate IFN- $\alpha$ and proinflammatory cytokines and chemokines [22]. Recently, our group has shown that IgG anti-NR2 glutamate receptor antibodies (anti-NR2) from SLE patients directly activated endothelial cells by the activation of NF- $\kappa \mathrm{B}$ signaling, resulting in the upregulation of adhesion molecules and 
TABLE 1: Cytokines in CSF of NPSLE.

\begin{tabular}{|c|c|c|c|c|}
\hline Cytokines & NPSLE & Control group & Authors & Year \\
\hline IL-1 & Incresed & None & Alcocer-Varela et al. [12] & 1992 \\
\hline IL-1 $\beta$ & Same & Neulogical symptoms without neurological diseases & Gilad et al. [13] & 1997 \\
\hline soluble IL-2R & Incresed & Neulogical symptoms without neurological diseases & Gilad et al. [13] & 1997 \\
\hline \multirow{9}{*}{ IL-6 } & Incresed & Non-NPSLE, HI & Jönsen et al. [14] & 2003 \\
\hline & Incresed & HI, neurocysticerosis & Jara et al. [15] & 1998 \\
\hline & Incresed & Non-NPSLE & Hirohata and Miyamoto [16] & 1990 \\
\hline & Incresed & Cerebral infarction & Hirohata and Hayakawa [17] & 1999 \\
\hline & Incresed & None & Alcocer-Varela et al. [12] & 1992 \\
\hline & Incresed & CNS inflammation, non-inflammatory CNS diseases & Tsai et al. [18] & 1994 \\
\hline & Incresed & Non-NPSLE & Trysberg et al. [19] & 2009 \\
\hline & Incresed & HI & Dellalibera et al. [20] & 2003 \\
\hline & Incresed & Non-NPSLE, SM, non-AID & Fragoso-Loyo [21] & 2007 \\
\hline \multirow{3}{*}{ IL-8 } & Incresed & Non-NPSLE & Trysberg et al. [19] & 2009 \\
\hline & Incresed & Non-NPSLE, SM, non-AID & Fragoso-Loyo [21] & 2007 \\
\hline & Incresed & MS, other AID & Santer et al. [22] & 2009 \\
\hline \multirow{3}{*}{ IL-10 } & Same & Non-NPSLE, HI & Jönsen et al. [14] & 2003 \\
\hline & Incresed & HI & Dellalibera et al. [20] & 2003 \\
\hline & Same & Non-NPSLE, SM, non-AID & Fragoso-Loyo [21] & 2007 \\
\hline \multirow{3}{*}{ TNF- $\alpha$} & Incresed & $\mathrm{HI}$ & Dellalibera et al. [20] & 2003 \\
\hline & Same & Non-NPSLE, SM, non-AID & Fragoso et al. [21] & 2007 \\
\hline & Same & Neulogical symptoms without neurological diseases & Gilad et al. [23] & 1997 \\
\hline IFN- $\gamma$ & Same & Non-NPSLE, SM, non-AID & Fragoso-Loyo et al. [21] & 2007 \\
\hline \multirow{3}{*}{ IFN- $\alpha$} & Incresed & Non-NPSLE, HI & Jönsen et al. [14] & 2003 \\
\hline & Incresed & Non-NPSLE & Winfield et al. [24] & 1983 \\
\hline & Incresed & Non-NPSLE & Shiozawa et al. [25] & 1992 \\
\hline
\end{tabular}

AID: autoimmune diseases, HI: healthy individuals, MS: multiple sclerosis, SM: septic meningitis.

cytokine production [29]. Further immunological studies are expected to show how autoantibodies in SLE patients work to promote the cytokine storm associated with the pathophysiology of NPSLE.

\section{Chemokines}

Chemokines in humans comprise more than 50 small (8to-10-kDa) heparin-binding proteins with 20 to 70 percent homology in amino acid sequences. Chemokines were originally identified by their chemotactic activity on bone marrow-derived cells $[30,31]$. They are classified into at least four families according to the location of their cysteine residues. The four chemokine groups are CC, C, CXC, and $\mathrm{CX} 3 \mathrm{C}$, where $\mathrm{C}$ is a cysteine and $\mathrm{X}$ any amino-acid residue, and their receptors are consequently classified as CCR, CR, CXCR, and CX3CR. The chemokine receptors are bound to the cell membrane through seven transmembrane helical segments coupled with a G-protein which transduces the intracellular signal. The two major subclasses include the $\mathrm{CC}$ chemokines where the cysteines are neighboring and the CXC chemokines where the cysteines are separated by one amino acid. The CXC chemokines mainly act on neutrophils and lymphocytes, whereas the CC chemokines mainly act on monocytes and lymphocytes without affecting neutrophils [32]. Lymphotactin, in the $\mathrm{C}$ chemokine family, is similar to members of both the CC and CXC chemokine families but lacks two of the four cysteine residues and is a potent attractant for $\mathrm{T}$ cells, but not for monocytes or neutrophils [33]. Fractalkine, in the CX3C family, is a cell-surface-bound protein, in which the first two cysteine residues are separated by three amino acids. Fractalkine has potent chemoattractant activity for $\mathrm{T}$ cells and monocytes [34]. One characteristic feature of chemokines is the redundancy of the system. Several chemokines bind to more than one receptor and the majority of chemokine receptors have multiple ligands leading to the generation of multiple pathways directing similar cellular responses. Until recently, chemokines have been named randomly, with no clear system being used. Some have been included in the same group as $\backslash$ interleukins (e.g., IL-8), while others have been given names describing a function, for example, macrophage chemoattractant proteins. In an attempt to clarify the confused and complex nomenclature associated with chemokines, the nomenclature of the chemokine system has been revised. The name referring to a specific biologic function has been replaced by the chemokine subfamily name followed by a number (e.g., MCP-1 is CCL2) $[35,36]$. In this review, chemokines are described with common 
TABLE 2: Chemokines and chemokine receptors in NPSLE.

\begin{tabular}{lcl}
\hline Chemokines & Chemokine receptors & Cell Types \\
\hline IL-8/CXCL8 & CXCR1 & Neutrophils, monocytes \\
IP-10/CXCL10 & CXCR3 & Th1 cells, mast cells, mesangial cells \\
fractalkine/CXCL1 & CX3CR1d & Macrophages, endothlial cells, smooth muscle cells \\
RANTES/CCL5 & CCR1 & Tcells, monocytes, eosinophils, basophils \\
RANTES/CCL5 & CCR3 & Eosinophils, basophils, mast cells, Th2 cells \\
MCP-1/CCL2 & CCR2 & Memory T cells, monocytes, immature dendritic cells \\
\hline
\end{tabular}

names as well as the revised names according to the new nomenclature. Several chemokines such as IL-8/CXCL8, IP10/CXCL10, fractalkine/CXCL1, RANTES/CCL5, and MCP$1 /$ CCL2 have been reported to be elevated in the CSF from patients with NPSLE (Table 2).

\subsection{As Biomarkers}

3.1.1. CC Chemokines. Monocyte chemoattractant protein (MCP)-1/CCL2 (a ligand of CCR2) can attract monocytes, T cells, NK cells, and basophils [7,37]. It is a high-affinity ligand for the CCR2 chemokine receptor, that is, constitutively expressed in monocytes but is expressed on lymphocytes only after stimultion by IL-2. Expression of CCR2 on monocytes can be downregulated by lipopolysaccharides. We and others have reported that CSF MCP-1/CCL2 levels are higher in NPSLE patients than in non-NPSLE patients $[7,21]$. In addition, we reported that levels of MCP-1/CCL2 decreased after immunosuppressive treatment. Furthermore, we compared the levels of MCP-1/CCL2 among various neuropsychiatric symptoms. However, as some patient groups have very few cases, we were unable to conclude which type of symptom was associated with the increase of CSF MCP1/CCL2 levels in our study [7].

(RANTES)/CCL5 (a ligand of CCR1, CCR3, and CCR5) is another CC chemokine which attracts monocytes, memory T cells and NK cells and is implicated in the pathophysiology of SLE, rheumatoid arthritis (RA), and multiple sclerosis (MS) [38]. Chemokine receptor CCR5 is preferentially expressed on Th1 lymphocytes and has been reported to have an important role in the pathogenesis of RA. It has been reported that systemic administration of a small molecular weight antagonist of CCR5, SCH-X, suppressed the development of collagen-induced arthritis (CIA) in a monkey model of RA [39]. We also provided evidence showing that systemic administration of TAK-779, a nonpeptide compound with a small molecular weight, inhibits the development of adjuvant-induced arthritis in rats [40]. Recent reports suggest that CSF levels of CCL5 are increased in NPSLE patients compared with non-NPSLE patients [21].

3.1.2. CXC Chemokines. IL-8/CXCL8 (a ligand of CXCR1 and CXCR2) was the first chemokine identified to be involved in leukocyte chemotaxis such as polymorphonuclear neutrophils and specific $\mathrm{T}$ cells $[41,42]$. There are several reports showing that IL-8/CXCL8 levels in CSF are elevated in NPSLE [21,43].

Interferon-gamma inducible protein-10 (IP-10)/CXCL10 (a ligand of CXCR3) is expressed and secreted by monocytes and fibroblasts following stimulation with IFN- $\gamma[29,44]$. IP-10/CXCL10 is a high-affinity ligand for the CXCR3 chemokine receptor which is mainly expressed on helper T1 (Th1) cells. The predominance of Th1 versus Th2 cells in NPSLE patients remains unresolved. We and others have reported that IP-10/CXCL10 was upregulated in the CNS fluid of NPSLE $[9,21]$.

3.1.3. $C$ and $C X 3 C$ Chemokines. The $\mathrm{C}$ chemokine family is represented by two chemokines, lymphotactin/XCL1, and SCM-1 $\beta / \mathrm{XCL} 2$, whereas the $\mathrm{CX}_{3} \mathrm{C}$ chemokine family contains only one member, called fractalkine/CX ${ }_{3}$ CL1 [47]. Fractalkine/CX $\mathrm{CX}_{3} \mathrm{CL} 1$ is synthesized by endothelial cells as a type 1 transmembrane protein which is then cleaved by proteolysis, possibly mediated by TNF- $\alpha$-converting enzyme (TACE) and ADAM 10, thereby yielding the soluble form of Fractalkine/CX ${ }_{3} \mathrm{CL} 1$ (sFKN). Fractalkine/CX ${ }_{3} \mathrm{CL} 1$ binds to a receptor known as CXCR1 signaling via the $\mathrm{G}$ protein pathway in NK cells, macrophages and a certain proportion of T cells. Fractalkine/CX ${ }_{3}$ CL1 plays important roles in the pathogenesis of RA by attracting proinflammatory cells, such as activated macrophages and $\mathrm{T}$ cells [48]. There is a report showing that levels of sFKN/s $\mathrm{CX}_{3} \mathrm{CL} 1$ were elevated in the CSF of NPSLE. In this report, both serum and CSF sFKN/s $\mathrm{CX}_{3}$ CL1 levels declined with treatment [45]. However, our group did not find a significant increase of sFKN/s $\mathrm{CX}_{3} \mathrm{CL} 1$ in CSF from NPSLE patients compared with that of nonNPSLE patients [46].

3.1.4. Ratio of Two Different Chemokine Levels. We reported that the IP-10/MCP-1 ratio is a useful marker to detect NPSLE [8]. In our study, the IP-10/MCP-1 ratio in the NPSLE group was significantly higher than that in the nonNPSLE group ( $P=.0000014$, Mann-Whitney's $U$ test). The discriminative ability (area under the curve) of each chemokine was 0.63111 (95\% confidence interval [95\% CI] 822.4064-7787.1975) (IP-10/CXCL10), 0.67626 ([95\% CI] 418.6142-1761.8262) (MCP-1/CCL2), and 0.82672 ([95\% CI] 1.222729-5.011448) (IP-10/MCP-1 ratio). There was no correlation between the levels of MCP-1 in CSF and that in $\operatorname{serum}(r=-1332, P=.2481)$ and the levels of IP-10 in CSF and that in serum $(r=-1445, P=.3842)$. There was 


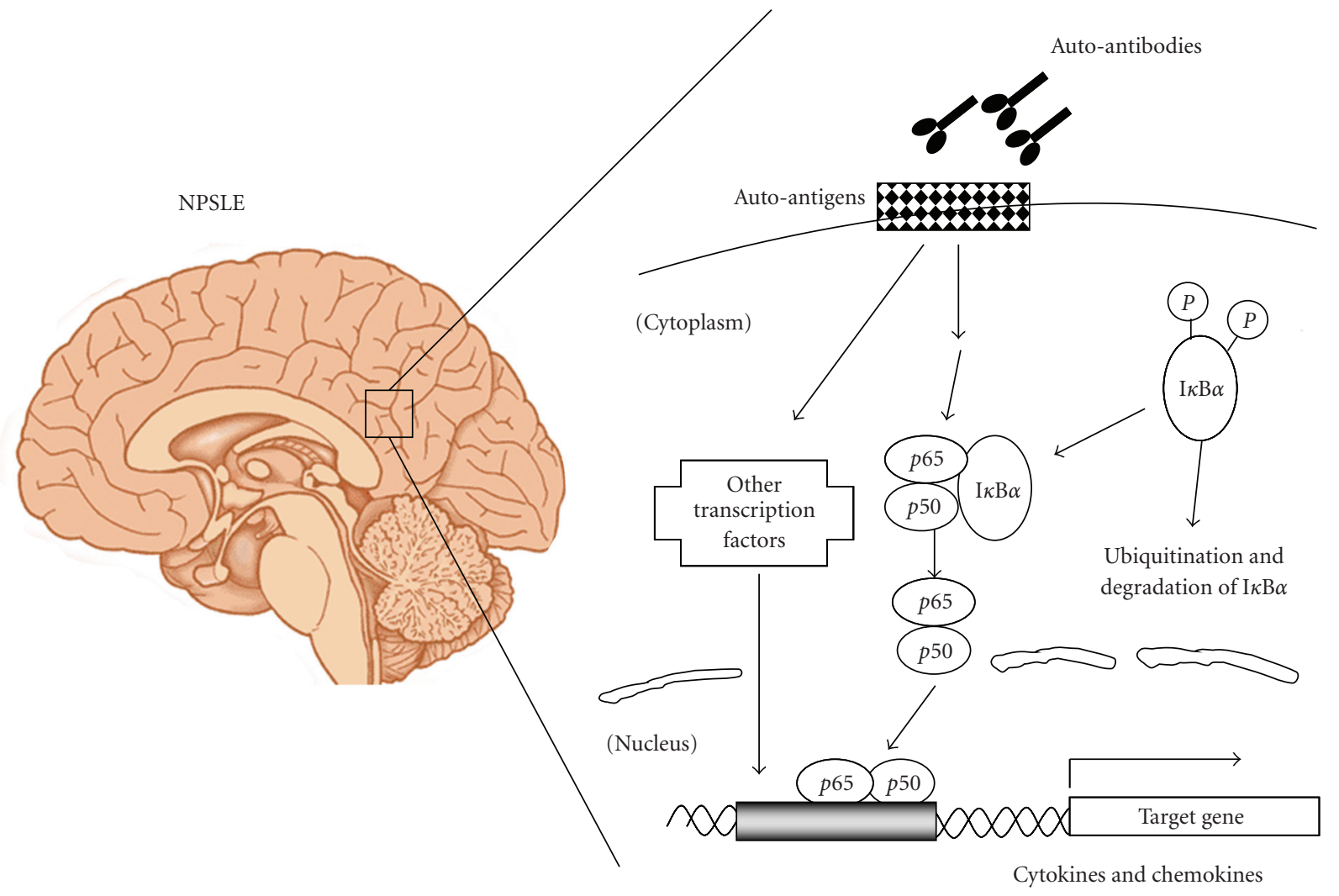

FIgURe 1

TABLE 3: CHemokines in CSF of NPSLE.

\begin{tabular}{|c|c|c|c|c|}
\hline Chemokines & NPSLE & Control group & Authors & Year \\
\hline \multirow{2}{*}{ MCP-1/CCL2 } & Increased & Non-NPSLE & Iikuni et al. [7] & 2006 \\
\hline & Increased & Non-NPSLE & Fragoso-Loyo [21] & 2007 \\
\hline RANTES/CCL5 & Increased & Non-NPSLE & Fragoso-Loyo [21] & 2007 \\
\hline \multirow{3}{*}{ IL-8/CXCL8 } & Increased & Non-NPSLE & Trysberg et al. [43] & 2003 \\
\hline & Increased & Non-NPSLE, SM, non-AID & Fragoso-Loyo [21] & 2007 \\
\hline & Increased & MS, other AID & Santer et al. [22] & 2009 \\
\hline IP-10/CXCL10 & Increased & Non-NPSLE & Okamoto et al. [9] & 2004 \\
\hline \multirow{2}{*}{ Fractalkine } & Increased & Non-NPSLE & Yajima et al. [45] & 2005 \\
\hline & Same & Non-NPSLE & Sato et al. [46] & 2006 \\
\hline IP-10/MCP-1 ratio & Increased & Non-NPSLE & Okamoto et al. [8] & 2006 \\
\hline
\end{tabular}

AID: autoimmune diseases, MS: multiple sclerosis, SM: septic meningitis.

also no correlation between the levels of MCP-1 and IL-6 $(r=-0.1229, P=.462)$, IL-8 $(r=-0.1585, P=.349)$, and IFN alpha $(r=-0.0818, P=.179)$, and between IP10 and IL-6 $(r=-0.1435, P=.384)$, IL-8 $(r=-0.1553$, $P=.332)$, and IFN alpha $(r=-0.1021, P=.213)$. We concluded that CSF IP-10/MCP-1 ratios are higher in NPSLE patients than in non-NPSLE patients and that this index is a useful diagnostic marker of NPSLE [8].

The summary of the reported results are shown in Table 3.
3.2. As Pathogenic Factors. There is growing evidence showing that chemokines not only recruit specific subsets of lymphocytes and inflammatory cells but also determine the type of immune response at the site of inflammation and that chemokines have pivotal roles in the development and progression of autoimmune disorders.

In the course of an immune response, distinct subsets of effecter $\mathrm{T}$ cells and regulatory $\mathrm{T}$ cells are generated in the lymphoid compartments after the differentiation of naive $\mathrm{T}$ cells under the influence of specific cytokines. 
Chemokines guide these T-cell subsets out of the lymphoid compartment and into sites of inflammation and infection. Before the commitment of antigen-specific T-cell subsets, innate T cells such as NKT and $\gamma \delta \mathrm{T}$ cells work as the first line of host defence and also are guided to the sites of inflammation by chemokines. Naive T cells differentiate into Th1 cells under the direction of the cytokine IL-12 and transcription factor T-bet. Th1 cells are characterized by the production of IFN- $\gamma$ and have a role in the protection against intracellular pathogens. On the other hand, IL-4 and the transcription factor GATA-3 are critical in the differentiation of naive $\mathrm{T}$ cells into Th2 cells. Th2 cells are characterized by the production of IL-4, IL-5, and IL- 13 which mediate protection against parasites and allergic responses. Th1 cells preferentially express CCR5, CXCR3, and CXCR6, whereas Th2 cells preferentially express CCR4 and CCR8.

We reported that CSF MCP-1/CCL2 and IP-10/CXCL10 levels are higher in NPSLE patients than in non-NPSLE patients, indicating possible involvement of this chemokine in the pathogenesis of NPSLE. The receptor of IP10/CXCL10, CXCR3 is predominantly expressed on natural killer cells and activated T cells, especially Th1 cells. On the other hand, the receptor of MCP-1/CCL2, CCR2 is expressed not only on activated $\mathrm{T}$ cells and natural killer cells but also on monocytes, basophils, and dendritic cells. CD4+ T cells populations that upregulate expression of the transcription factor RORgt can differentiated into IL-17 producing CD4+ $\mathrm{T}$ cells (Th17 cells) that differ in phenotype and function from Th1 and Th2 cells. Th17 cells are thought to protect against bacteria and fungi and these cells are also involved in the pathogenesis of autoimmne diseases [44]. Interestingly, CCR2 is expressed on a subpopulation of Th17 cells which produce a large amount of IL-17 but little IFN- $\gamma$ [49]. These results implicate the differential contribution of both CXCR3 and CCR2 signaling in the pathogenesis of NPSLE, especially on effector T cells such as Th1, Th2, and Th17 cells.

\section{Conclusion}

Although a large number of studies have been performed, the precise pathophysiology of NPSLE is not completely understood. As we presented here, various cytokines and chemokines are highly expressed in the brain of NPSLE patients, and it is believed that these small molecules have important roles in the pathogenesis of NPSLE. However, the molecular mechanisms by which these molecules work in the course of the development of NPSLE have not yet been completely revealed. Cytokines and chemokines are expressed by the stimulation of NF- $\kappa \mathrm{B}$ signaling and signal transduction pathways involving other trancription factors [50]. As mentioned above, our group has shown that IgG antiNR2 glutamate receptor antibodies (anti-NR2) from SLE patients direct NF- $\kappa \mathrm{B}$ signaling in endothelial cells, resulting in the upregulation of adhesion molecules and cytokine production. Therefore, autoantibodies which are characteristic features of SLE bind to corresponding autoantigens on the cell surface and these interactions may stimulate signaling cascades, resulting in the activation of certain transcription factors (Figure 1). This activation of signal transduction pathways involving these transcription factors might activate transcription and expression of cytokines and chemokines, resulting in a cytokines/chemokines storm and the development of NPSLE pathophysiology. Further molecular studies are required to prove this proposed mode of action for cytokines and chemokines. In addition, cytokines and chemokines are considered to be therapeutic targets in NPSLE. As most of the cytokines and chemokines involved in NPSLE have pleiotrophic roles in other biological processes, inhibition of these cytokines and chemokines might invite unexpected side effects in vivo. Cooperative contribution of both clinical studies and molecular biological studies is required for the development of ideal therapeutic strategies against NPSLE.

\section{References}

[1] "The American College of Rheumatology nomenclature and case definitions for neuropsychiatric lupus syndromes. ACR Ad Hoc Committee on Neuropsychiatric Lupus Nomenclature," Arthritis and Rheumatism, vol. 42, no. 4, pp. 599-608, 1999.

[2] B. Bresnihan, "CNS lupus," Clinics in Rheumatic Diseases, vol. 8, no. 1, pp. 183-195, 1982.

[3] R. L. Brey, S. L. Holliday, A. R. Saklad et al., "Neuropsychiatric syndromes in lupus: prevalence using standardized definitions," Neurology, vol. 58, no. 8, pp. 1214-1220, 2002.

[4] H. Ainiala, J. Loukkola, J. Peltola, M. Korpela, and A. Hietaharju, "The prevalence of neuropsychiatric syndromes in systemic lupus erythematosus," Neurology, vol. 57, no. 3, pp. 496-500, 2001.

[5] E. Trysberg, H. Carlsten, and A. Tarkowski, "Intrathecal cytokines in systemic lupus erythematosus with central nervous system involvement," Lupus, vol. 9, no. 7, pp. 498-503, 2000.

[6] E. Svenungsson, M. Andersson, L. Brundin et al., "Increased levels of proinflammatory cytokines and nitric oxide metabolites in neuropsychiatric lupus erythematosus," Annals of the Rheumatic Diseases, vol. 60, no. 4, pp. 372-379, 2001.

[7] N. Iikuni, H. Okamoto, T. Yoshio et al., "Raised monocyte chemotactic protein-1 (MCP-1)/CCL2 in cerebrospinal fluid of patients with neuropsychiatric lupus," Annals of the Rheumatic Diseases, vol. 65, no. 2, pp. 253-256, 2006.

[8] H. Okamoto, N. Iikuni, S. Kamitsuji, T. Yoshio, S. Minota, and N. Kamatani, "IP-10/MCP-1 ratio in CSF is an useful diagnostic marker of neuropsychiatric lupus patients," Rheumatology, vol. 45, no. 2, pp. 232-234, 2006.

[9] H. Okamoto, Y. Katsumata, K. Nishimura, and N. Kamatani, "Interferon-inducible protein 10/CXCL10 is increased in the cerebrospinal fluid of patients with central nervous system lupus," Arthritis and Rheumatism, vol. 50, no. 11, pp. 37313732, 2004.

[10] M. Aringer, W. B. Graninger, G. Steiner, and J. S. Smolen, "Safety and efficacy of tumor necrosis factor $\alpha$ blockade in systemic lupus erythematosus: an open-label study," Arthritis and Rheumatism, vol. 50, no. 10, pp. 3161-3169, 2004.

[11] A. Rahman and D. A. Isenberg, "Systemic lupus erythematosus," New England Journal of Medicine, vol. 358, no. 9, pp. 929939, 2008. 
[12] J. Alcocer-Varela, D. Aleman-Hoey, and D. Alarcon-Segovia, "Interleukin-1 and interleukin-6 activities are increased in the cerebrospinal fluid of patients with CNS lupus erythematosus and correlate with local late T-cell activation markers," Lupus, vol. 1, no. 2, pp. 111-117, 1992.

[13] R. Gilad, Y. Lampl, Y. Eshel, V. Barak, and I. Sarova-Pinhas, "Cerebrospinal fluid soluble interleukin-2 receptor in cerebral lupus," British Journal of Rheumatology, vol. 36, no. 2, pp. 190193, 1997.

[14] A. Jönsen, A. A. Bengtsson, O. Nived et al., "The heterogeneity of neuropsychiatric systemic lupus erythematosus is reflected in lack of association with cerebrospinal fluid cytokine profiles," Lupus, vol. 12, no. 11, pp. 846-850, 2003.

[15] L. J. Jara, L. Irigoyen, M. J. De Ortiz, B. Zazueta, G. Bravo, and L. R. Espinoza, "Prolactin and interleukin-6 in neuropsychiatric lupus erythematosus," Clinical Rheumatology, vol. 17, no. 2, pp. 110-114, 1998.

[16] S. Hirohata and T. Miyamoto, "Elevated levels of interleukin6 in cerebrospinal fluid from patients with systemic lupus erythematosus and central nervous system involvement," Arthritis and Rheumatism, vol. 33, no. 5, pp. 644-649, 1990.

[17] S. Hirohata and K. Hayakawa, "Enhanced interleukin-6 messenger RNA expression by neuronal cells in a patient with neuropsychiatric systemic lupus erythematosus," Arthritis and Rheumatism, vol. 42, no. 12, pp. 2729-2730, 1999.

[18] C.-Y. Tsai, T.-H. Wu, S.-T. Tsai et al., "Cerebrospinal fluid interleukin-6, prostaglandin E2 and autoantibodies in patients with neuropsychiatric systemic lupus erythematosus and central nervous system infections," Scandinavian Journal of Rheumatology, vol. 23, no. 2, pp. 57-63, 1994.

[19] E. Trysberg, H. Carlsten, and A. Tarkowski, "Intrathecal cytokines in systemic lupus erythematosus with central nervous system involvement," Lupus, vol. 9, no. 7, pp. 498-503, 2000.

[20] R. Dellalibera-Joviliano, M. L. Dos Reis, F. D. Q. Cunha, and E. A. Donadi, "Kinins and cytokines in plasma and cerebrospinal fluid of patients with neuropsychiatric lupus," Journal of Rheumatology, vol. 30, no. 3, pp. 485-492, 2003.

[21] H. Fragoso-Loyo, Y. Richaud-Patin, A. Orozco-Narváez et al., "Interleukin-6 and chemokines in the neuropsychiatric manifestations of systemic lupus erythematosus," Arthritis and Rheumatism, vol. 56, no. 4, pp. 1242-1250, 2007.

[22] D. M. Santer, T. Yoshio, S. Minota, T. Möller, and K. B. Elkon, "Potent induction of IFN-alpha and chemokines by autoantibodies in the cerebrospinal fluid of patients with neuropsychiatric lupus," Journal of Immunology, vol. 182, no. 2, pp. 1192-1201, 2009.

[23] R. Gilad, Y. Lampl, Y. Eshel, V. Barak, and I. Sarova-Pinhas, "Cerebrospinal fluid soluble interleukin-2 receptor in cerebral lupus," British Journal of Rheumatology, vol. 36, no. 2, pp. 190193, 1997.

[24] J. B. Winfield, M. Shaw, L. M. Silverman, et al., "Intrathecal IgG synthesis and blood-brain barrier impairment in patients with systemic lupus erythematosus and central nervous system dysfunction," American Journal of Medicine, vol. 74, no. 5, pp. 837-844, 1983.

[25] S. Shiozawa, Y. Kuroki, M. Kim, S. Hirohata, and T. Ogino, "Interferon-alpha in lupus psychosis," Arthritis and Rheumatism, vol. 35, no. 4, pp. 417-422, 1992.

[26] S. Hirohata, Y. Kanai, A. Mitsuo, Y. Tokano, and H. Hashimoto, "Accuracy of cerebrospinal fluid IL-6 testing for diagnosis of lupus psychosis. A multicenter retrospective study. NPSLE Research Subcommittee," Clinical Rheumatology, vol. 28, no. 11, pp. 1319-1323, 2009.
[27] A. George-Chandy, E. Trysberg, and K. Eriksson, "Raised intrathecal levels of APRIL and BAFF in patients with systemic lupus erythematosus: relationship to neuropsychiatric symptoms," Arthritis Research and Therapy, vol. 10, no. 4, article R97, 2008.

[28] Y. Katsumata, M. Harigai, Y. Kawaguchi et al., "Diagnostic reliability of cerebral spinal fluid tests for acute confusional state (delirium) in patients with systemic lupus erythematosus: interleukin 6 (IL-6), IL-8, interferon- $\alpha$, IgG index, and Qalbumin," Journal of Rheumatology, vol. 34, no. 10, pp. 20102017, 2007.

[29] T. Yoshio, S. Yamazaki, H. Okamoto, et al., "IgG antiNR2 glutamate receptor antibodies (anti-NR2) from patients with systemic lupus erythematosus (SLE) as causative autoantibodies of damage to the blood-brain barrier (BBB)," in Proceedings of the ACR Annual Scientific Meeting, 2009.

[30] I. F. Charo and R. M. Ransohoff, "Mechanisms of disease: the many roles of chemokines and chemokine receptors in inflammation," New England Journal of Medicine, vol. 354, no. 6, pp. 610-621, 2006.

[31] T. Iwamoto, H. Okamoto, Y. Toyama, and S. Momohara, "Molecular aspects of rheumatoid arthritis: chemokines in the joints of patients," FEBS Journal, vol. 275, no. 18, pp. 44484455, 2008.

[32] A. D. Luster, "Mechanisms of disease: chemokineschemotactic cytokines that mediate inflammation," New England Journal of Medicine, vol. 338, no. 7, pp. 436-445, 1998.

[33] G. S. Kelner, J. Kennedy, K. B. Bacon et al., "Lymphotactin: a cytokine that represents a new class of chemokine," Science, vol. 266, no. 5189, pp. 1395-1399, 1994.

[34] J. F. Bazan, K. B. Bacon, G. Hardiman et al., "A new class of membrane-bound chemokine with a CX3C motif," Nature, vol. 385, no. 6617, pp. 640-642, 1997.

[35] A. Zlotnik and O. Yoshie, "Chemokines: a new classification system and their role in immunity," Immunity, vol. 12, no. 2, pp. 121-127, 2000.

[36] K. Bacon, M. Baggiolini, H. Broxmeyer, et al., "Chemokine/chemokine receptor nomenclature," Journal of Interferon \& Cytokine Research, vol. 22, pp. 1067-1068, 2002.

[37] P. Loetscher, M. Seitz, I. Clark-Lewis, M. Baggiolini, and B. Moser, "Monocyte chemotactic proteins MCP-1, MCP-2, and MCP-3 are major attractants for human $\mathrm{CD} 4^{+}$and $\mathrm{CD} 8^{+} \mathrm{T}$ lymphocytes," FASEB Journal, vol. 8, no. 13, pp. 1055-1060, 1994.

[38] T. J. Schall, K. Bacon, K. J. Toy, and D. V. Goeddel, "Selective attraction of monocytes and T lymphocytes of the memory phenotype by cytokine RANTES," Nature, vol. 347, no. 6294, pp. 669-671, 1990.

[39] M. P. M. Vierboom, P. J. Zavodny, C.-C. Chou et al., "Inhibition of the development of collagen-induced arthritis in rhesus monkeys by a small molecular weight antagonist of CCR5," Arthritis and Rheumatism, vol. 52, no. 2, pp. 627-636, 2005.

[40] H. Okamoto and N. Kamatani, "A CCR-5 antagonist inhibits the development of adjuvant arthritis in rats," Rheumatology, vol. 45 , no. 2, pp. 230-232, 2006.

[41] B. Moser and P. Loetscher, "Lymphocyte traffic control by chemokines," Nature Immunology, vol. 2, no. 2, pp. 123-128, 2001.

[42] M. Baggiolini, A. Walz, and S. L. Kunkel, "Neutrophilactivating peptide-1/interleukin 8 , a novel cytokine that activates neutrophils," Journal of Clinical Investigation, vol. 84, no. 4, pp. 1045-1049, 1989. 
[43] E. Trysberg, K. Nylen, L. E. Rosengren, and A. Tarkowski, "Neuronal and astrocytic damage in systemic lupus erythematosus patients with central nervous system involvement," Arthritis and Rheumatism, vol. 48, no. 10, pp. 2881-2887, 2003.

[44] S. K. Bromley, T. R. Mempel, and A. D. Luster, "Orchestrating the orchestrators: chemokines in control of T cell traffic," Nature Immunology, vol. 9, no. 9, pp. 970-980, 2008.

[45] N. Yajima, T. Kasama, T. Isozaki et al., "Elevated levels of soluble fractalkine in active systemic lupus erythematosus: potential involvement in neuropsychiatric manifestations," Arthritis and Rheumatism, vol. 52, no. 6, pp. 1670-1675, 2005.

[46] E. Sato, N. Iikuni, T. Yoshio, S. Minota, N. Kamatani, and H. Okamoto, "Soluble fractalkine in the cerebrospinal fluid of patients with neuropsychiatric lupus," Annals of the Rheumatic Diseases, vol. 65, no. 9, pp. 1257-1259, 2006.

[47] L. Stievano, E. Piovan, and A. Amadori, "C and CX3C chemokines: cell sources and physiopathological implications," Critical Reviews in Immunology, vol. 24, no. 3, pp. 205-228, 2004.

[48] G. Murphy, N. Caplice, and M. Molloy, "Fractalkine in rheumatoid arthritis: a review to date," Rheumatology, vol. 47, no. 10, pp. 1446-1451, 2008.

[49] W. Sato, T. Aranami, and T. Yamamura, "Cutting edge: human Th17 cells are identified as bearing CCR $2^{+} \mathrm{CCR} 5^{-}$phenotype," Journal of Immunology, vol. 178, no. 12, pp. 7525-7529, 2007.

[50] H. Okamoto, T. P. Cujec, H. Yamanaka, and N. Kamatani, "Molecular aspects of rheumatoid arthritis: role of transcription factors," FEBS Journal, vol. 275, no. 18, pp. 4463-4470, 2008. 


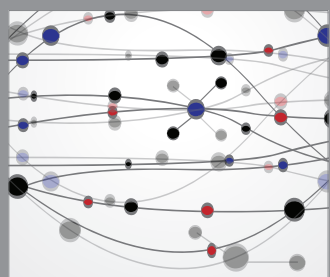

The Scientific World Journal
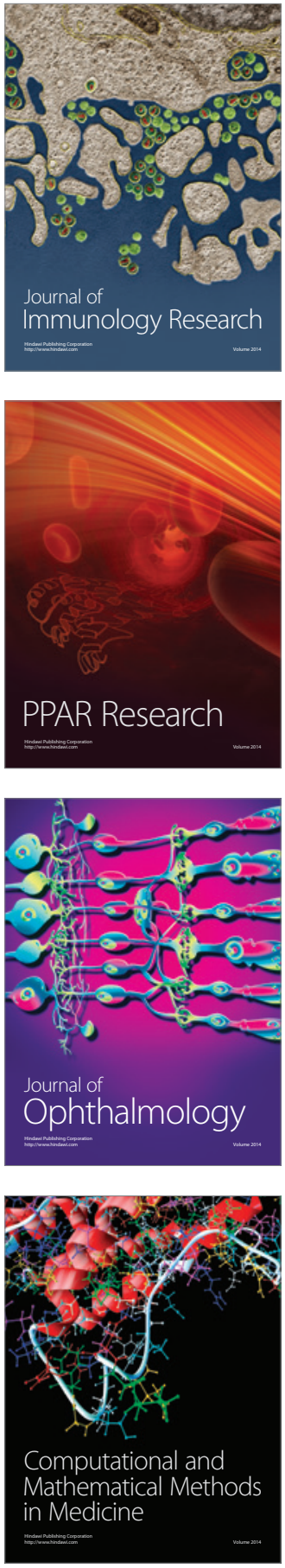

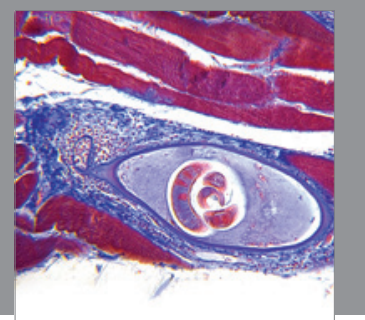

Gastroenterology

Research and Practice
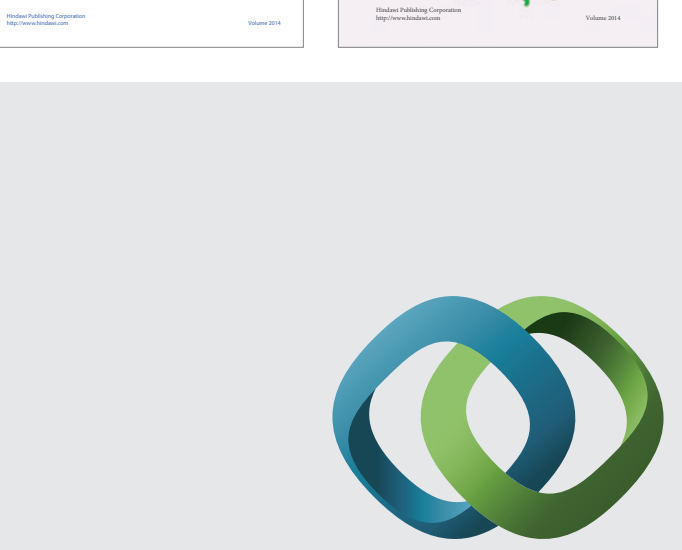

\section{Hindawi}

Submit your manuscripts at

http://www.hindawi.com
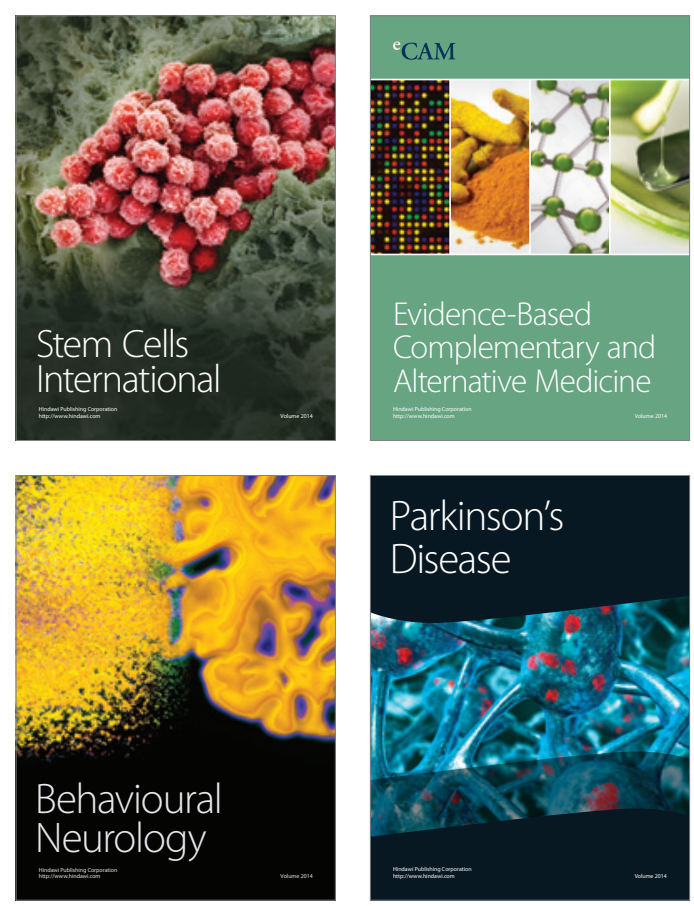

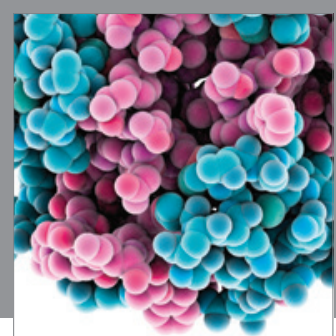

Journal of
Diabetes Research

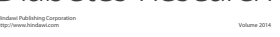

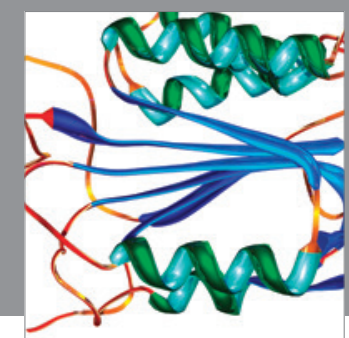

Disease Markers
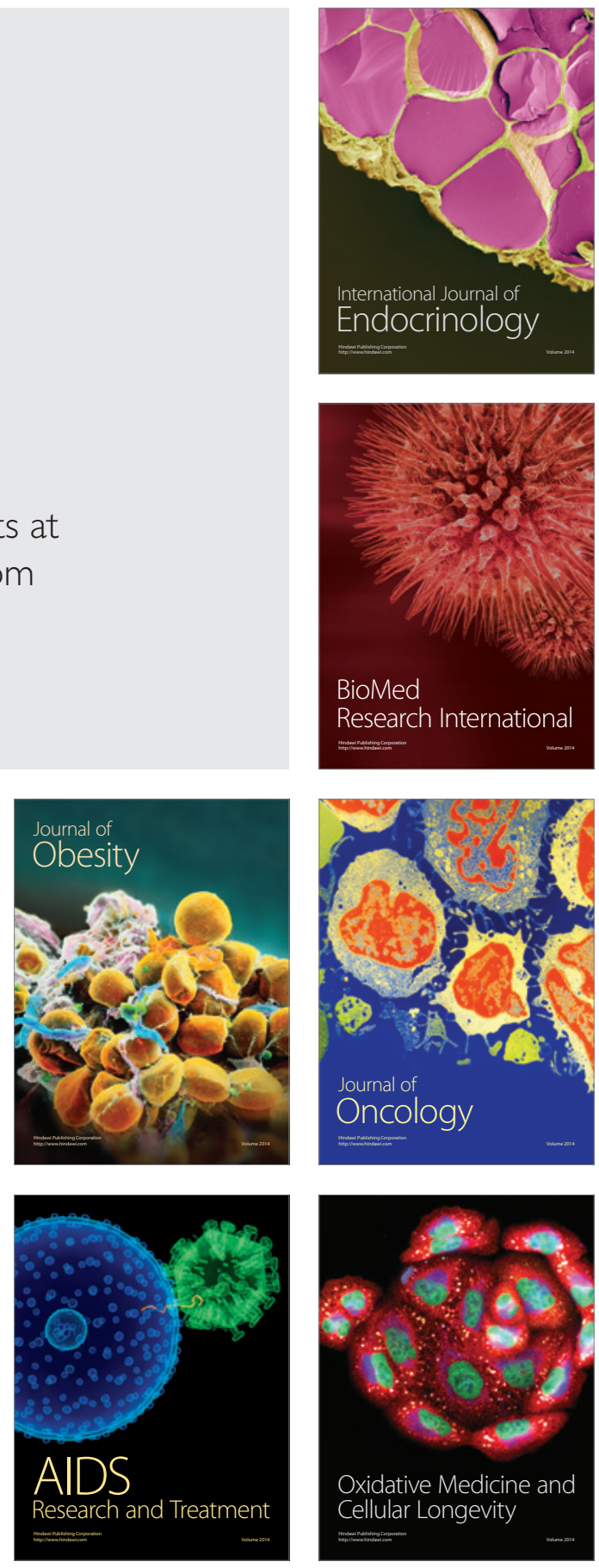